\title{
Tony Manero de Pablo Larraín (2008) ou l'avènement du vide comme esthétique de l'échec
}

\section{Dominique Casimiro}

\section{(2) OpenEdition}

1 Journals

\section{Édition électronique}

URL : https://journals.openedition.org/cher/12194

DOI : $10.4000 /$ cher.12194

ISSN : 2803-5992

\section{Éditeur}

Presses universitaires de Strasbourg

\section{Édition imprimée}

Date de publication : 30 juin 2012

Pagination : 103-115

ISBN : 978-2-35410-046-9

ISSN : 1968-035X

\section{Référence électronique}

Dominique Casimiro, «Tony Manero de Pablo Larraín (2008) ou l'avènement du vide comme esthétique de l'échec », reCHERches [En ligne], 8 | 2012, mis en ligne le 21 février 2022, consulté le 23 février 2022. URL : http://journals.openedition.org/cher/12194; DOI : https://doi.org/10.4000/cher.12194

\section{(c) (i) (ㅇ)}

Ce(tte) œuvre est mise à disposition selon les termes de la Licence Creative Commons Attribution Pas d'Utilisation Commerciale - Partage dans les Mêmes Conditions 4.0 International. 


\title{
Tony Manero de Pablo Larraín (2008) ou l'avènement du vide comme esthétique de l'échec
}

\author{
Dominique Casimiro \\ Université de la Sorbonne Nouvelle
}

e blanc est la couleur d'une énigme. «Si 'blanc' est un concept qui se
rapporte uniquement à une surface visuelle, pourquoi donc n'y a-t-il pas
un concept de couleur apparenté à 'blanc' qui se rapporterait à quelque chose
de transparent?», s'interroge Wittgenstein (50). «Au cinéma, notifie-t-il, il
est souvent possible de voir les événements du film comme s'ils se situaient
derrière l'écran et que celui-ci fût transparent comme un panneau de verre.
[...] Or nous ne sommes pas tentés cependant de nommer ce panneau
de verre blanc transparent». Le blanc est censé ne rien laisser voir de ce
qui se situe au-delà de lui, pas plus, du reste, qu'il ne participe à l'illusion
de la profondeur: «Si toutes les couleurs devenaient plus blanches, alors
l'image perdrait de plus en plus de profondeur» (50). Pourtant, cette opacité
nécessaire du blanc n'est pas liée à sa matière: «On ne peut pas dire non plus
que le blanc soit essentiellement la propriété d'une surface (visuelle). Car on
pourrait imaginer que le blanc se produisît simplement comme un éclat, ou
comme la couleur d'une flamme» (43). En effet, une autre caractéristique du
«blanc» est sa luminosité: «Si un fantôme m'apparaissait dans la nuit, il se
pourrait qu'il émît une faible lueur blanchâtre; mais s'il m'apparaissait gris,
alors il faudrait que la lumière parût venir de quelque part ailleurs» (55).
Ainsi, d'être conçu comme la couleur même de la «surface», le blanc est à
la fois une couleur qui masque et dissimule, mais sans pour autant signifier
l'absence: c'est en désignant sa présence qu'elle voile le monde. Le blanc
serait aussi une proposition de lumière - sinon la lumière elle-même - et 
cela, quel que soit l'éclairage auquel sa surface se trouve soumise: «le papier que voici est plus ou moins clair selon les endroits, note encore Wittgenstein; mais puis-je dire qu'il est blanc à certains endroits et gris en d'autres? - Certes, si je le peignais, je mélangerais en tout cas un gris pour les endroits plus sombres» (30).

Si le blanc échappe donc au réel, s'il est une matière mentale, la surface qu'il définit peut être indifféremment interprétée comme vide - au sens où tout événement ou manifestation visuelle effective a toujours lieu en dehors d'elle (le blanc exclut par principe la trace ou la maculature: leur apparition le divise sans l'entamer) - ou pleine - puisque sa couleur est l'indice autosuffisant d'une présence impénétrable, qu'il s'agisse de la surface d'un objet ou de la luminosité de cette surface - ou encore comme une lumière "faisant surface" à sa façon, telle la flamme d'un feu ou d'une bougie. Cependant, que l'on tende à l'interpréter plutôt d'une manière ou d'une autre, le blanc est toujours et simultanément vide et plein, signe avantcoureur d'un invisible à ce point inconcevable et dense qu'il peut être perçu comme une absence, mais aussi don de lumière total et immédiat, spectacle d'emblée absolu de tous les possibles réalisables. Telle est l'énigme soulevée par Wittgenstein.

Telle est également l'énigme soulevée par le réalisateur chilien Pablo Larraín dans le film que nous prétendons interroger ici - Tony Manero (2008) - et qui écrit en images «l'inquiétante étrangeté» d'un homme, Raúl Peralta, qui, dans le Santiago du Chili de 1978, alors que le régime dictatorial instauré par Pinochet en est à ses heures les plus sombres, n'a qu'une obsession: voir et revoir, jusqu'à l'ivresse, La Fièvre du samedi soir (1977) de John Badham. Véritable cauchemar éveillé d'une force rare, le film de Pablo Larraín nous livre une vision âpre et glaçante du Chili d'hier et de l'amnésie qui le frapperait encore aujourd'hui. Au moyen d'un jeu fécond d'oppositions chromatiques (entre le noir et le blanc, dans un premier temps) et lumineuses, le spectateur découvrira la tentative d'existence frustrée d'un homme qui, au cours de l'émission télévisée Festival de la Una, déclinera son identité civile en ces termes:

Enrique MALUENDA (presentador del programa) - ¿Cómo está? ¡Muy buenas tardes! ¿Cuál es su nombre?

Raúl - Raúl Peralta Paredes O.

Enrique MALUENDA - ¿O? Bueno, muy bien... Paredes. Vieja pared del arrabal... Es bonito este tango, ¿a usted le gustan los tangos?

Raúl - Sí.

Enrique MALUENDA - Ah... qué bueno, qué bueno. ¿A qué se dedica 
usted? ¿En qué trabaja?

Raúl - A esto.

Enrique MALUENDA - ¿Pero qué es "a esto"? ¿A bailar?

Raúl - Sí.

Enrique MALUENDA - ¿Ah sí? Ya ya...

Déguisé en "Tony Manero», Raúl est une première illustration des thèses de Wittgenstein sur le blanc : comme ce mystérieux patronyme " $\mathrm{O}$ » le laisse entendre, sous ce costume blanc se cache une vérité ontologique: en temps de dictature, l'homme se révèlerait tel qu'il est, à savoir une coquille vide, une non-existence, un antihéros, un loser fini. Si le blanc est, en ce début de film, la couleur visible par excellence puisqu'elle ne se laisse pas traverser et qu'elle est elle-même source de lumière sur la scène du programme chilien Festival de la Una, ce blanc n'est qu'une illusion. Derrière cette couleur se cacherait du noir. Creusons donc ici la lumière et le blanc, le strass et les paillettes et découvrons ce qui se cache sous le costume de "Tony Manero». Comme l'a si élégamment mis en musique Enrique Maluenda, Raúl est un homme presque vieux et pas très beau; néanmoins il a dans sa tête un rêve et un seul: être élu sosie national de Tony Manero et gagner ainsi un poncho de la marque Lindo, un paquet de sauce Pastitalia, un blender et, surtout, une somme d'argent suffisamment rondelette pour pouvoir survivre dans le Chili léthargique et tétanisé de 1978. Oui, mais n’est pas Tony Manero qui veut...

Pablo Larraín nous offre la radiographie d'un des représentants de la frange a-humaine et minable de la société chilienne de l'époque, une radiographie qui a pour but esthétique et ontologique de nous donner

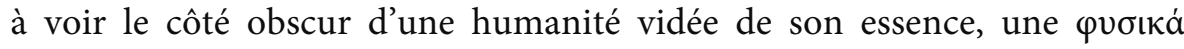
physiká de l'être humain qui, comme nous le conclurons, n'est là que pour révéler l'échec de l'être humain dans son entreprise (vaine?) d'être humain. Derrière ce blanc maculé du costume de Tony Manero s'offre donc au réalisateur Pablo Larraín la possibilité de relever le défi suivant: comment faire vivre le vide au cinéma afin de montrer le vide de la condition humaine d'un homme, "Raúl Peralta Paredes O.», citoyen chilien ordinaire sous le Chili du général Pinochet?

Parce qu'il se refuse à un cinéma de l'évidence et du trop-plein, Pablo Larraín choisit ici de filmer le vide incarné par Raúl comme l'expression d'un échec inéluctable, fils lui-même du néant dans lequel est plongé le Chili de Pinochet, et qui emplit ses habitants de doutes, de peurs et surtout de frustrations. Or, ce défi esthétique relève d'un paradoxe fécond: 
interroger le cinéma dans son rapport au vide, c'est aborder une notion qui met profondément en jeu l'art cinématographique et, pour ainsi dire, le provoque dans ce que l'on a, à la suite d'André Bazin, appelé son «essence réaliste». Que ce soit au nom d'un positivisme myope, d'une ontologie suspecte ou d'un illusionnisme consolateur, le cinéma a été pensé et perçu (et continue sans doute à l'être) en termes d'effet de présence et d'impression de plénitude. Le cinéma ne posséderait donc pas ce pouvoir de néantisation qu'un Stéphane Mallarmé ou un Kazimir Malevitch ont su tirer du langage poétique et pictural. Ce ne serait alors pas un carré blanc sur fond blancqui pourrait resplendir sur l'écran, mais une présence pleine et prosaïque, enlisée dans l'opacité de l'hic et nunc, et limitée à sa seule immédiateté. Et cependant, cette esthétique du vide semble la seule capable de mettre en lumière et en sons des êtres minables, comme "Raúl Peralta Paredes O.», des êtres mis sous vide.

Je me propose donc de réfléchir sur cette esthétique paradoxale au travers de la leçon d'anatomie du raté monstrueux que nous propose Pablo Larraín dans cette œuvre filmique particulière, Tony Manero, qui devrait acquérir peut-être, en fin de parcours, valeur de paradigme de cette courte trajectoire cinématographique d'un réalisateur chilien prometteur. Aussi, m'efforceraije de répondre aux questions suivantes: comment Pablo Larraín parvient-il à représenter ces irreprésentables, à représenter le vide a-humain au moyen du vide cinématographique? Comment ces ersatz d'humanité que sont «Raúl Peralta Paredes O.» et sa clique de danseurs du dimanche peuvent-ils contaminer ici les images mêmes qui les désignent, les images mêmes qui les représentent et les mettent en scène? Serait-ce parce que dans les plis de la rhétorique filmique ils se sentent chez eux? Ou comment le cinéma peut filmer l'échec sans sombrer lui-même dans l'échec.

Tony Manero est un film au casting sobre construit autour d'un noyau de quatre irréductibles ratés: Raúl, que nous connaissons, sa maîtresse occasionnelle Cony, sa fille Pauli, et Wilma, la tenancière de la pension miteuse des bas-quartiers de Santiago où logent et dansent le samedi soir ces protagonistes. Tous forment, à n'en pas douter, des purs produits de la logique de terrorisme d'État instituée par Augusto Pinochet, mais tous en sont également des victimes: ils sont des ratés de la nature, ils sont des monstres à visage humain. Rappelons que Cony n'hésitera pas à dénoncer sa propre fille, qui, prise de «la fièvre du samedi soir», s'est un peu trop rapprochée de Raúl. Rappelons également que Raúl Peralta Paredes O. est tout simplement un psychopathe, nourri de ses frustrations, qui n'hésite pas à tuer, dès lors qu'il sent poindre une possible contrariété. Lui qui 
semble sans passé, économe en paroles et en émotions (il ne profère que des phrases simples, récite froidement des répliques de La Fièvre du samedi soir ou insulte), lui qui n'hésite pas à se précipiter sur une vieille dame que des jeunes viennent d'agresser pour la détrousser encore plus facilement, rêve pourtant d'un avenir à la mesure de son idolâtrie: gagner le prochain concours "Yo soy... Tony Manero». En attendant ce jour, l'homme se jettera à corps perdu dans un projet frisant la schizophrénie: être un autre en devenant un personnage qui n'existe que sur les écrans de cinéma. Son adoration peut, au demeurant, être comprise au sens chrétien du terme. Impassible face au monde qui l'entoure, le regard de Raúl devient fiévreux dès qu'il se retrouve devant cet écran de cinéma où le corps de John Travolta se déhanche. La mise en abîme est ici évidente, mais néanmoins féconde: miroir aux alouettes, l'écran de cinéma est ce qui permettra à Raúl de remplir ce « $\mathrm{O}$ » vide en se construisant en double de Tony Manero. Mais ce miroir est aussi un miroir non déformant mais grossissant: seul face à son idole, Raúl est un raté qui répète inlassablement - et laborieusement -, l'anglais de répliques qu'il pense connaître par cœur. D'ailleurs, Pauli s'interrogera après un étrange soliloque en anglais de Raúl : ¿¿Él sabe inglés?».

Pour plus d'efficacité, Pablo Larraín ira jusqu'à copier-coller la scène culte de La Fièvre du samedi soir de John Badham où, filmé en contreplongée, John Travolta alias Tony Manero se prépare devant son miroir. À la défaveur de cette pâle imitation qu'est Raúl Peralta Paredes O, bien évidemment. Comme semblent y inviter le noir et blanc du costume de John Travolta, Raúl ne sera que le négatif de Tony Manero, son double en raté. Et en tant que tel, il sera condamné à se demander durant tout le film si la fermeture du pantalon de Tony Manero possède un ou deux boutons.

L'adjectif "pâle» est à prendre au pied de la lettre puisque le travail chromatique de Pablo Larraín construit, autour de ce degré zéro de la couleur qu'est la notion d'incolore, un homme qui n'est qu'une enveloppe vide, que le négatif d'un être humain. Le blanc, nous l'avons rappelé dans notre introduction, est une couleur révélatrice et ici, la révélation du vide inhérente à l'être humain se fait par une couleur qui n'est $n i$ le blanc ou plus le blanc, mais l'incolore. Si le vide était une couleur, celle-ci serait en effet l'incolore. Tout comme le vide semble difficile à sonder, définir cette notion d'incolore est un exercice malaisé, tant la plupart des explications ou des interprétations proposées par les différents dictionnaires sont hésitantes, imprécises, voire contradictoires. Néanmoins, l'historien des couleurs Michel Pastoureau (145-153) relève et valide la proposition du répertoire le 
plus riche et le plus savant consacré au lexique qu'est le Trésor de la langue française. Voici ce que notifie le dictionnaire; nous conviendrons alors que cette définition pourrait s'appliquer à Raúl:

Incolore: 1. (au sens propre): qui n’a pas de couleur bien déterminée, qui n'a pas de couleur propre, qui manque de teint (syn.: limpide, transparent, pâle); 2. (au sens figuré): qui manque de netteté ou d'éclat, qui est dépourvu d'expression, d'originalité, de personnalité (syn.: morne, monotone, terne).

Enfin, si l'échec était un objet, il serait en définitive ce slip que Raúl exhibe fréquemment et dont la blancheur - plus que douteuse - serait à l'image de ses cheveux grisonnants qu'il est obligé de teindre artisanalement. La photographie de Tony Manero utilise fréquemment la technique efficace du contre-jour qui parvient à donner une forme - certes floue - à l'informe. Pablo Larraín ne fait ici que donner un corps à l'échec que l'affiche du film synthétise également : grâce à la lumière projetée par une boule à facettes de pacotille (elle sera construite par Raúl à partir d'un ballon de football sur lequel il aura collé au préalable des morceaux de miroir cassé), le regardeur ne distingue plus d'un être humain qu'un bras, un front et le haut d'un crâne; le reste du personnage semble plongé dans cette obscurité qui inondera constamment à l'écran Raúl, lorsque celui-ci bascule dans le côté obscur et animal de son personnage à chacun de ses crimes. Si l'incolore est la couleur du vide, le noir sera donc celle de l'animalité; je vous renvoie à la scène finale où Raúl se prépare pour le concours - et pour son plus beau crime - paré de son plus beau slip noir et coiffé de sa plus belle chevelure artificiellement brune. Entre l'incolore (qui peut être le blanc, comme toutes les couleurs à la fois, souligne Michel Pastoureau) et le noir, le raté pourra également adopter une enveloppe caméléonesque, comme le laissent comprendre à plusieurs reprises les scènes où Raúl, véritable charognard, sort de sa tanière pour dépouiller le corps encore chaud des dernières victimes de la dictature pinochetiste.

À plusieurs reprises, faisant trembler la caméra de Pablo Larraín, Raúl cours vers un destin, lui aussi de plus en plus noir. Événement et avènement d'une bestialité à peine refoulée, le concours "Yo soy... Tony Manero» sera alors l'occasion pour Raúl de commettre son crime le plus atroce: tuer le sosie gagnant et sa femme. Cette scène, qui constitue la scène finale du film, n'apparaîtra à l'écran que suggérée par un fondu enchaîné qui finira sur un rectangle noir. Quand l'esprit, essayant à son tour d'échapper à cette spirale qu'est l'échec, apprend que le salut ne peut venir d'autrui en temps de dictature, il se laisse définitivement happer par le vide. Cet écran noir est 
la cristallisation de la béance de solitude qui s'ouvre sous les pieds de Raúl dès les premiers plans du film, lorsque l'angoisse et la folie s'emparent de lui, et du Chili. Mais le néant est parfois moins effrayant que la vie... et la mort plus rassurante.

En somme, Pablo Larraín parvient à donner une couleur à une notion qui, comme Raúl, manque de teint, de netteté, d’originalité, de personnalité. À partir d'une déclinaison du blanc "wittgensteinien ", le cinéaste écrit en couleurs incolores l'a-humanité de l'homme en temps de guerre. Pour ce faire, il utilise également une pellicule dont la subtilité du grain est essentielle à la vie de ce raté: le réalisateur ayant opté pour un grain de pellicule très prononcé, l'effet de saleté visuelle complète cette esthétique du vide et de l'incolore, en donnant davantage de chair au manque d'humanité de cet homme. Mi-documentaire, mi-pictural, associé à des séquences filmées caméra à l'épaule, ce grain donne corps à l'échec qui n'est plus seulement une forme informe à contempler, mais une forme dotée des qualités tactiles d'une matière rugueuse, un relief dont il faut épouser les courbes, les creux ou les arêtes.

Pablo Larraín poursuit sa leçon d'anatomie en soumettant le corps de ses protagonistes à une véritable inspection panoptique. Délaissant le souci de la forme et de l'art pour satisfaire le seul désir de révéler le vide de ces enveloppes a-humaines remplies de frustrations, le réalisateur exhibe jusqu'aux parties les plus intimes et les mieux cachées de ces personnages. Attention, aucun dispositif voyeuriste, aucune chambre tapissée de miroirs ou hérissée de webcams, ne viendra surprendre dans ce film le secret du nu puisque, selon la filmographie de Pablo Larraín, la nudité intégrale n'existe pas. Serait-il montré simultanément sous toutes ses coutures, le corps resterait fermé à l'inspection panoptique; à moins d'être ouvert et transformé en cadavre, (comme cela sera le cas dans le dernier film de Pablo Larraín, Santiago 73 Post mortem [2010]); à moins d'être objet d'une leçon d'anatomie, comme cela est le cas dans Tony Manero, le corps du raté ne peut apparaître à l'écran qu'au moyen d'enchaînements rapides de plans serrés et fragmentés. Les scènes les plus significatives sont, évidemment, celles où l'impuissance sexuelle de Raúl est magnifiée par une succession de plans traduisant l'impossibilité d'une plénitude sexuelle.

La presque nudité des corps chez Pablo Larraín est le signe de leur faiblesse et de leur échec, qui devient très vite détresse, puisque leur peau, à vif, ne parviendra jamais à rencontrer une autre peau. Échange vital, la caresse - toujours unilatérale dans Tony Manero - ne sera que la confirmation que 
le toucher ne rapprochera ni ne confondra jamais deux peaux. Écriture de l'échec dont le tracé ne parvient pas à éveiller les sens, ni à frayer sur la peau les voies du sens. L'échec est donc un tatouage primordial que possèdent tous les protagonistes de ce film. Il est un énième vide entre les corps comme l'écrit le parallélisme des corps inertes de Raúl et Pauli, après une tentative vaine et frustrée de rapprochement sexuel. Comme l'écrit la scène où ces deux corps se retrouvent allongés l'un contre l'autre (presque peau contre peau), les corps auraient pu être à la fois touchants et touchés, sujet et objet de la sensation. En se dépouillant des oripeaux de ce qu'il conviendrait d'appeler un déguisement (dans cette scène, Raúl est déguisé en danseur et, pour ce faire, a délaissé sa traditionnelle chemise incolore pour une d'un éclatant et enivrant bordeaux), les amants ne se dévoilent paradoxalement jamais l'un à l'autre. Dépouillé de sa peau invisible, le presque nu intégral du raté ne fait de lui qu'un écorché: livré tout entier à l'inspection anatomique du réalisateur et du spectateur, il n'est plus touchant ni touché, mais violent (et/ou violé). Et ceci, indépendamment du fait qu'il porte une chemise éclatante, ou une fleur - rouge aussi - dans les cheveux.

Cony et Raúl découvrent cependant ensemble une dimension inconnue: le vaste territoire de leur échec. Entre eux s'ouvre, en effet, un nouveau vide dessiné par des corps parallèles, un vide qui leur appartient et auquel ils appartiennent. Tout l'art du cinéaste Pablo Larraín consiste à cristalliser l'avènement de ce vide au travers de la séparation physique des corps et au travers d'une métaphysique des chairs usées. Face au corps rouillé de Raúl, face au sexe congestionné de Raúl, la sentence de la nudité ne se fera pas attendre; elle sera rendue par Cony en ces termes: «Tenemos que hacerlo rapidito porque el tiempo va pasando. El Tony, el de la película no envejece nunca. En cambio usted se está poniendo viejito. [...] Se le hincha un poco nomás. Pero no se le para». La rencontre de deux épidermes aimantés par le désir aurait pu créer une sorte de champ magnétique où auraient circulé des flux d'énergie traversant les corps pour les unir l'un à l'autre, et les rendre perméables aux influx de la vie universelle. Mais il n'en sera rien. Le contact le plus superficiel inaugurera malheureusement une profondeur abyssale: le vide de l'être humain. Interface entre l'intérieur et l'extérieur, la peau devient poreuse à l'échec.

Derrière cette séparation définitive des corps, Pablo Larraín définit également une éthique de la nudité, qui ne doit pouvoir être touchée qu'à condition d'être touchante. Or la nudité du raté n'est pas touchante; elle est salissante. Le nu est l'objet de la pulsion scopique, qui l'isole et l'immobilise 
pour mieux le saisir; l'échec se révèle alors dans l'épreuve du nu et du toucher non réciproque (Pauli préfèrera, rappelons-le, se masturber aux côtés de Raúl plutôt que de succomber entièrement à ses charmes) : l'échec n'est pas un objet, mais l'expérience non partagée de deux sujets, la frustration. De ce fait, il est latéral, et non frontal. Il est mouvant et émouvant, alors que le nu suppose l'immobilité du modèle et l'impassibilité de l'artiste. Il déploie autour de lui une mouvance qui déborde toute figure et communique avec le fond, corps subtil qui participe de l'illusion collective, selon Pablo Larraín, d'une chair du monde.

À cette illusion collective d'un possible bonheur contribue également un personnage qu'on ne verra jamais à l'écran mais qui, cependant, apparaîtra dans le générique final, au côté des personnages: la bande originale. Les Bee Gees hantent évidemment Tony Manero puisqu'ils sont un rappel de La Fièvre du samedi soir, mais ce groupe n'est rien à côté du groupe pop chilien, Frecuencia Mod, dont cinq chansons résonnent hors champ. Véritable institution au Chili, ce groupe a été savoureusement croqué par la journaliste Marisol García en des termes très élogieux:

Chile no estaba para fiestas a fines de los años setenta, pero la música de Frecuencia Mod logró, incluso bajo el severo toque de queda, reproducir a escala local la fiebre disco. El grupo fue un trío vocal [cuyas] cuidadas armonías vocales y prolijos arreglos legaron un sonido que aún suena elegante, muy por encima de similares esfuerzos bailables de la época. Aunque en su momento Frecuencia Mod defendió una música de aparente evasión, es imposible no reconocer su valor pionero para el pop chileno de la más alta factura (132).

Bien évidemment, il nous faut aller bien au-delà de ces considérations purement subjectives, et considérer l'apport de cette bande originale à la mise en sons, cette fois-ci, de l'échec. "La pensée se fait dans la bouche", nous disait Tristan Tzara: elle nait du contact entre l'air et les organes ultra-sensibles de la phonation, dont nous pouvons, si nous y prêtons attention, percevoir les mouvements subliminaux. Mais que faire lorsqu'on est incapable de penser, lorsque, comme Raúl Peralta, on est incapable de toute «danse laryngo-buccale» (Spire: 76) ? Écouter en boucle Cállate (ya no me mientas).

Es muy tarde ya para soñar

(Es muy tarde ya para soñar)

Muchas veces te lo repetí.

Calla por favor, 
No sigas no, no, no, no, no...

Que nuestra barca naufragó

Se bercer d'illusion. Combler ce vide par des vibrations sirupeuses à la fois sonores et tangibles, dont les échos (matérialisés par cette négation «no» qui résonne dans le vide et qui riment avec l'oxyton "naufragó») nous confirment que la seule représentation possible de l'échec, au cinéma, passerait par un hors-champ sonore et par l'incolore. Car le vide que signifie l'échec ne semble pouvoir se manifester que dans la hantise de ce qui est absent, tourné vers un dehors qu'il dramatise, vers une présence confirmée dans une zone d'ombre du champ, et qu'il permet de tenir pleinement absente en désignant son retrait, en mettant en scène son im-présentation. En cela, la scène du show à l'américaine de Raúl lors du concours est saisissante: l'ellipse entre les plans 34 et 35 figure le vide opéré en Raúl par un retour glaçant à la réalité. Par ce jeu d'exacerbation du manque à voir que ce plan manquant instaure au cœur de la représentation, et par la dynamique de déploiement imaginaire que ce même plan oblige (nous attendions cette scène depuis le début du film, et Pablo Larraín choisit de la montrer sous forme fragmentée et elliptique, nous condamnant, par là même, à remplir ce vide fécond), l'ellipse figure un dehors du champ qu'elle met en branle.

Qu'il s'expose dans le plan à travers la nudité suprématiste du champ vide, ou qu'il s'impose de façon latente comme effet à travers les interstices qu'ouvre au cœur du plan - mais surtout entre ces deux plans - un mode d'exposition fragmenté, déconnecté, décadré et elliptique, le vide participerait d'une logique de suspension du sens. Même s'il opère comme signe absent, neutre ou opaque qui cristallise l'a-humanité de ces êtres à une absence, ce plan vide excède toute représentation avec laquelle il établit un mystérieux contact: contact absolu et irrationnel d'un dedans non totalisable (régi par une succession d'images fragmentées, lacunaires et coupées du monde) et d'un dehors invisible (hors-champ absolu, non actualisable) qui, animé et aspiré par la force du vide, pénètre par toutes les jointures laissées béantes par le cinéaste.

Alors que dans le cinéma classique, l'image cinématographique avait, pour reprendre les termes de Gilles Deleuze, «essentiellement un hors-champ qui renvoyait d'une part à un monde extérieur actualisable dans d'autres images, d'autre part à un tout changeant qui s'exprimait dans l'ensemble des images associées» (L'image-temps, Cinéma 2: 233), dans le style de Pablo Larraín, l'image laissée en blanc, comprimée sur elle-même et isolée du monde extérieur se prolonge au-delà de son propre cadre et entre dans 
un rapport d'actualisation avec le hors-champ. Celui-ci ne désigne plus «ce qui existe ailleurs, à côté ou autour [...] mais [...] témoigne d'une présence plus inquiétante, dont on ne peut même plus dire qu'elle existe, mais plutôt qu'elle insiste ou subsiste, un Ailleurs plus radical, hors de l'espace et du temps homogènes" (L'image-mouvement, Cinéma 1:30). Ce hors-champ absolu éprouve dans la construction du cinéaste chilien comme l'assomption de quelque chose qui perfore le film, d'une force du désir frustré qui opère une fracture interne au sein même de l'univers perçu et se donne à voir dans l'espace du plan ou dans l'entre deux plans sous la modalité virtuelle du vide: un vide "qui n'est plus une part motrice de l'image et qu'elle franchirait pour continuer mais qui est la mise en question radicale de l'image» (L'image-temps : 235) confrontée à l'irruption du transcendant... un point de contact irrationnel avec un dehors qui accomplit le passage d'un «ensemble fermé que l'on fragmente à un tout spirituel ouvert que l'on crée ou recrée» (L'image-mouvement: 165).

Je terminerai par ce constat et cette interrogation: et si l'empreinte métonymique de Raúl qu'estl'ellipse (chromatique, lumineuse, ontologique... mais aussi ce « $\mathrm{O}$ » de son étrange identité civile) n'était là que pour nous frustrer également? Nous serions alors tentés de crier au scandale et de déplorer l'échec de la tentative de représentation de l'échec de Pablo Larraín. Or, il n'en est rien, il me semble. Loin d'être réductible à un thème (le thème de l'absence...), à une figure (la figure du hors-champ, du champ vide...), à un procédé (le décadrage...), à une forme isolable et identifiable dans l'image ou dans le film, le vide, pensé dans sa double valeur représentative et productive, opère davantage comme une modalité de la représentation qui «affecte» l'image ou le film tout entier sans être «cernable» ou décomposable. Il se définit comme un concept-limite (tout comme Raúl est un conceptlimite) qui met en question le cinéma dans son rapport au monde, au réel, au sens, au visible et à son propre langage, en le confrontant à une dimension (celle du vide, du dehors, de l'hétérogène, de l'opaque, du non raccord...) qu'il a longtemps refoulée et à laquelle il a fini par s'abandonner, vertigineusement, jusqu'à s'exténuer dans une logique cinématographique entièrement déterminée par une esthétique du vide. Charge au spectateur de combler ces vides. Charge à la société chilienne d'aujourd'hui de ne pas oublier les échecs du passé. Ces échecs qui, comme le « $\mathrm{O}$ » de «Raúl Paredes Peralta O.» résonnent dans la chanson de Frecuencia Mod, Cállate (ya no me mientas). 
Dans le jeune cinéma de Pablo Larraín, la figure du vide opère donc essentiellement comme un support d'échange fonctionnel qui met l'image cinématographique en rapport avec un dehors diégétique et relatif qui la prolonge (le hors-champ habité par la diégèse) ou l'informe (le champ vide hanté par le champ aveugle). Par un jeu d'exacerbation d'un manque à voir qu'elle instaure au cœur de la représentation et par la dynamique de déploiement imaginaire, de la diégèse vers un dehors du champ qu'elle met en branle, la logique d'évidement du plan participe d'une stratégie d'animation, de diégétisation de l'échec qui expose l'image aux régimes métonymiques de l'empreinte, de l'ellipse (suggestive ou frustrante): régimes de l'incolore, de la présence suspendue, en attente ou en part d'actualisation. Car le vide ne semble pouvoir se manifester que dans la hantise de ce qui est absent, tourné vers un dehors qu'il dramatise, vers une présence confirmée dans une zone d'ombre du champ ou dans le hors-champ, et qu'il permet de tenir pleinement absente en désignant son retrait, en mettant en scène son im-présentation.

Filmer l'échec semble donc possible. Pablo Larraín nous démontre ici que le processus qui régit le cinéma moderne et le conduit à se confronter de plein fouet au vide s'inscrit bien dans une logique de la finitude, de la réduction, de la résorption, dans ce que Serge Dancy appelle «le bouclage», «bouclage de la pulsion scopique», mais aussi des pulsions narratives et symboliques. Ce régime de déflation visuelle, fictionnelle et sémantique que s'est imposé finalement le cinéma dès la fin de la guerre (et le cinéma chilien dès la fin de la dictature du général Augusto Pinochet) a marqué profondément la courte histoire de la modernité cinématographique dans la diversité de ses manifestations et de ses partis pris esthétiques au point d'ouvrir la voie à des logiques cinématographiques du vide fécondes: le cinéma de Pablo Larraín s'attache à désigner le vide et surtout à faire de cette désignation l'un des énoncés principaux de son discours et l'un des enjeux essentiels de son écriture; un cinéma qui dit le vide mais aussi qui pratique l'effet de vide (filmer le vide); un cinéma qui n'échoue pas dans son entreprise de filmer l'échec. 


\section{Bibliographie}

Deleuze, Gilles, 1983, L’image-mouvement, Cinéma 1, Paris, Éditions de Minuit, coll. "Critique».

Deleuze, Gilles, 1985, L'image-temps, Cinéma 2, Paris, Éditions de Minuit, coll. «Critique».

Freud, Sigmund, 1971, "L'inquiétante étrangeté», Essais de psychanalyse appliquée, Paris, Gallimard, coll. «Idées».

García, Marisol, 1996, Historia de la música chilena, Santiago de Chile, Austral.

Larraín, Pablo, Tony Manero, 2008, scénario de Pablo Larraín, Alfredo Castro et Mateo Iribarren, NetworkDVD, 2009.

Pastoureau, Michel, 2010, La couleur des souvenirs, Paris, Le Seuil.

Spire, André, 1986, Plaisir poétique et plaisir musculaire, Paris, José Corti.

Wittgenstein, Ludwig, 1983, Remarques sur les couleurs, Paris, Trans-EuropRepress. 